\title{
$A B C$-transporter activity and autocrine eicosanoid-signaling are required for germ cell migration a basal chordate
}

\author{
Susannah H. Kassmer* ${ }^{* 1}$, Delany Rodriguez ${ }^{1}$, Anthony DeTomaso ${ }^{1}$ \\ ${ }^{1}$ Molecular, Cellular and Developmental Biology, University of California, Santa Barbara, \\ CA USA. \\ Susannah.kassmer@lifesci.ucsb.edu
}

\begin{abstract}
:
In the colonial ascidian Botryllus schlosseri, long-lived germline stem cells (GSCs) migrate to new germline niches as they develop during repetitive cycles of asexual reproduction. $A B C$-transporters are involved in the export of lipid-signaling molecules, but their roles in germ cell migration are poorly understood. Here, we show that in Botryllus, abcc1 and abcb1 are highly expressed in germ cells, and inhibition of ABCtransporter activity leads to failure of germ cell migration. Phospholipase A2 (PLA2) produces arachidonic acid, which is further metabolized to eicosanoid signaling molecules. In humans, 12-lipoxygenase (LOX) metabolizes arachidonic acid to12Hydroxyeicosatetraenoic acid (12-S-HETE), which stimulates migration of mammalian cancer cells and smooth muscle cells. We show that PLA2 and LOX activity are required for germ cell migration. A potential homolog to the human receptor for 12-S-HETE, $B S g p r 31$, is expressed in germ cells. 12-S-HETE rescues migration towards S1P in the presence of inhibitors of ABCC1, ABCB1, PLA2 or LOX, and a gradient of 12-S-HETE enhances chemotaxis towards S1P and stimulates motility. We conclude that 12-SHETE is a secondary chemoattractant exported by ACB-transporters that is required for migration of germ cells towards S1P. We also find that in the presence of S1P, detection of an 12-S-HETE gradient initiates an autologous positive feedback loop that may sustain migration. This is the first report of an eicosanoid-signaling molecule regulating germ cell migration.
\end{abstract}

Key words: ABC-transporter, eicosanoid, germ cell, migration, 12-S-HETE, lipoxygenase 


\section{Introduction:}

Cell migration is a fundamental process of development and maintenance of multicellular organisms and mediates tissue organization, organogenesis, immune response and homeostasis ${ }^{1}$. Regulation of cell migration requires a complex interplay of signaling cascades that influence cell adhesion, polarization and cell motility. Temporal-spatial cues are tightly controlled, as dysregulation of cell migration can have catastrophic consequences for the organism, including developmental defects and cancer.

In many species, germ cells that are specified during embryonic development need to migrate across the embryo to reach the somatic gonad, where they will develop into eggs and testes ${ }^{2}$. Germ cell migration is studied in a variety of organisms, and many features are widely conserved. Most germ cells undergo an active migration toward their somatic niche that is often be guided by a combination of attractive and repulsive cues ${ }^{3}$.

In addition to being cell membrane components, research in the last two decades has shown that many lipids, now termed "bioactive lipids", have critical cell signaling functions ${ }^{4}$. Some lipid classes such as lysophospholipids (including sphingosine-1phosphate), eicosanoids (e.g. prostaglandins), and endocannaboids can signal through receptors in the cell membrane ${ }^{4}$. These lipids activate G-protein coupled receptors (GPCRs) of the $\mathrm{Gi}, \mathrm{Gq}$, and $\mathrm{G} 12 / 13$ type can be activated by gradients of bioactive lipids and thus influence cell polarity. Bioactive lipids associated with cell polarity include lysophospholipids (LPLs) and phosphatidylinositolphosphates (PIPs). LPLs are bioactive lipids that can be generated by hydrolytic cleavage of fatty acid from glycerophospholipids, a reaction catalyzed by phospholipases. Distinct phospholipases cleave off either one of the two (PLA1 and PLA2) fatty acid residues. The biosynthesis of eicosanoids is initiated by the activation of PLA2, leading to the release of arachidonic acid. Arachidonic acid is metabolized to a variety of eicosanoid signaling molecules such as thromboxanes, and leukotrienes ${ }^{4}$. 
So far, very little is known about the roles of bioactive lipids in germ cell migration. A potential role for lipid signaling in germ cells was first discovered in Drosophila, in which mutations in the lipid phosphate phosphatases (LPP), wunen and wunen2, disrupt directed migration of germ cells to the gonad ${ }^{3,5}$, but the ligand has not been identified. However, this mechanism appears to be conserved, as LPPs also repel germ cells away from nearby somites in the zebrafish ${ }^{6}$. Recently, our group discovered that migration of germ cells is directed by the lysophospholipid Sphingosine-1-phosphate (S1P) in the colonial ascidian Botryllus schlosseri ${ }^{7}$.

Botryllus is a unique model to study germline biology, because an individual does not grow by increasing in size, but rather by a lifelong, recurring asexual budding process (called blastogenesis) during which entire bodies including all somatic and germline tissues. are regenerated de novo (Figure $1 \mathrm{~A}, \mathrm{~B}$ ). This results in a constantly expanding colony of genetically identical individuals, called zooids, which are linked by a common extracorporeal vasculature (Figure 1).

The best understood regenerative process in Botryllus occurs in the germline. Like most metazoans, Botryllus sets aside a population of PGCs early in embryogenesis that are responsible for gametogenesis for the life of the organism ${ }^{8}$. However, unlike most model organisms, Botryllus retains a population of mobile, self-renewing, lineage-restricted adult germline stem cells (GSCs) that retain pluripotency for life of the individual ${ }^{9}$. During the weekly development of new zooids, a new germline niche is formed, and GSCs migrate from the old germline niche to the new. A subset of these GSCs settle, differentiate into gametes (zooids are hermaphrodites and make sperm and eggs), while others self-renew and migrate to the next generation. Asexual development is synchronized throughout the colony, and migration occurs over a defined $48 \mathrm{~h}$ period as GSCs leave the primary bud niche and enter the secondary bud niche via the vasculature joining the two (Figure 1A, B, C). During this window, GSCs are also found in the colony vasculature and ampullae. GSC migration is controlled by a S1P gradient, which is synthesized in the new niche and binds to the GPCR S1PR1on migrating GSCs ${ }^{7}$ (Figure $1 \mathrm{C}$ ). 
A

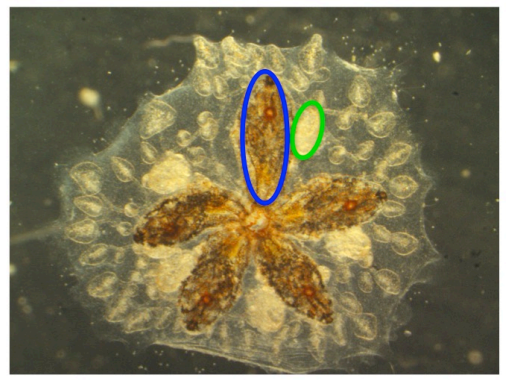

B

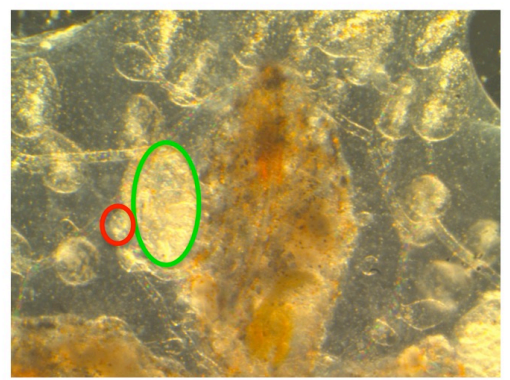

C

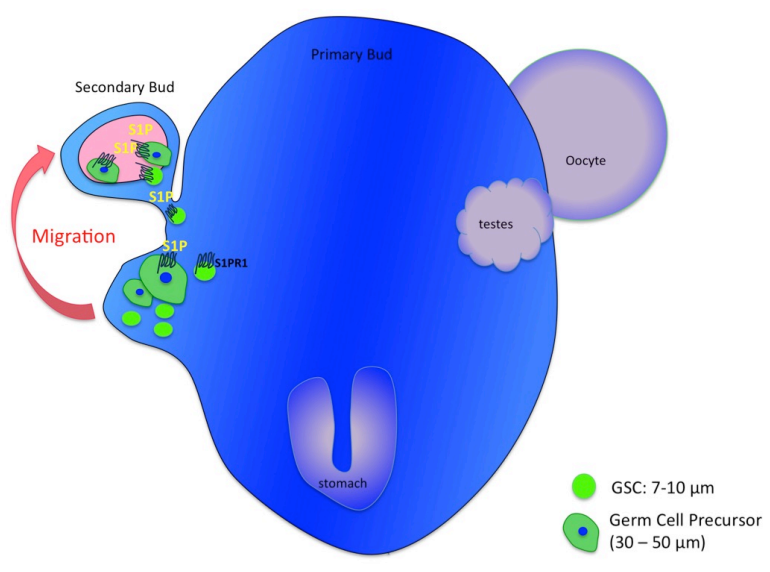

Figure 1: Morphology of Botryllus schlosseri colony, germ cell migration to secondary buds.

$\mathrm{A}$ and $\mathrm{B}$ : colony morohology and asexual reproduction. All individual bodies within the colony are embedded in an extracellular matrix known as the tunic and share an extracorporeal vasculature. Adult zooids (blue outline) asexually reproduce by giving rise to primary (green outline) and secondary buds (red outline). C: Migration of germ cells to secondary buds. Secondary buds begin as small protrusions of the body wall of primary buds, and later form a closed double vesicle. This vesicle later grows and undergoes invaginations and tissue differentiation, completing development into the adult form. Germline stem cells and (GSC, $7-10 \mu \mathrm{M})$ and germ cell precursors $(30-50 \mu \mathrm{M})$ migrate from the primary bud into the secondary bud at the time when the double vesicle forms. When the double vesicle is fully formed, germ cells have completed migration. Migration into the secondary bud is directed by a chemotactic gradient of sphingosine-1phosphate, which is secreted within the secondary bud and detected by sphingosine-1phosphate-receptor-1 expressed by the migrating germ cells (Kassmer et al 2015).

The ATP binding cassette $(A B C)$ are transport proteins that are conserved in all phyla from prokaryotes to humans ${ }^{10}$. $A B C$ transporters shuttle a variety of hydrophobic lipophilic compounds across the cell membrane in an ATP-dependent manner, including bioactive lipids such as S1P, leukotrienes and prostaglandins ${ }^{11}$. Some ABC transporters play roles in cell migration. In Drosophila, a germ cell attractant is geranylgeranylated 
and secreted by mesodermal cells in a signal peptide-independent manner through an ABCB transporter of the MDR family ${ }^{12}$. Chemotaxis of human dendritic cells to CCL19 requires stimulation with the exogenous leukotriene $\mathrm{C} 4$, an eicosanoid transported out of the cell via $A B C C 1^{13}$.

Cells responding to a primary chemoattractant can secrete secondary chemoattractants that increase the robustness of the primary chemotactic response ${ }^{14,15}$. Neutrophils release leukotriene $B_{4}\left(L_{T B}\right)$ to enhance their chemotactic response to the inflammatory cue fMLP. Binding of fMLP to cell surface receptors initiates leukotriene biosynthesis by stimulating the conversion of arachidonic acid (AA) to $\mathrm{LTB}_{4}$. LTB 4 is released as a secondary chemoattractant, and stimulates neutrophil motility through its interaction with its cognate receptor BLT1. The $\mathrm{LTB}_{4}$ is packaged in exosomes, which are secreted in a polarized fashion to the region of the cell with the highest fMLP concentration, setting up a gradient along the cell itself. Failure to form or detect the secondary chemoattractant causes an impaired chemotactic response ${ }^{15}$.

In the present study, we show that activity of $A B C C 1$ and $A B C B 1$ is required for directed migration of Botryllus germ cells in shallow gradients of the primary chemoattractant S1P in vitro and for homing towards developing germline niches in vivo. We identify the lipoxygenase-product 12-S-HETE as a secondary chemoattractant that enhances chemotaxis towards shallow gradients of S1P. Botryllus germ cells express a homolog of the human 12-S-HETE receptor GPR31. We conclude that shallow gradients of S1P induce activation of LOX and production and ABC-transporter-mediated export of 12-SHETE, resulting in autocrine stimulation of chemotaxis.

\section{Results:}

\section{Vasa-positive cells express $A B C C 1$ and $A B C B 1$}

We have shown previously that vasa-positive germline stem cells can be prospectively isolated from the blood of Botryllus schlosseri by flow cytometry, using a mononclonal antibody against Integrin-alpha-6 (IA6) ${ }^{7}$. By quantitative real time PCR, abcc1 and abcb1 mRNA are highly enriched in IA6+ cells, with abcb1 is expressed at higher levels than abcc1 (6.2 fold enrichment vs 2.2 fold enrichment, Figure 2A). We confirmed 
expression of abcc1 and $a b c b 1$ in vasa-positive cells migrating to secondary buds (Arrows, Figure 2B) by fluorescent in situ hybridization (FISH).

A

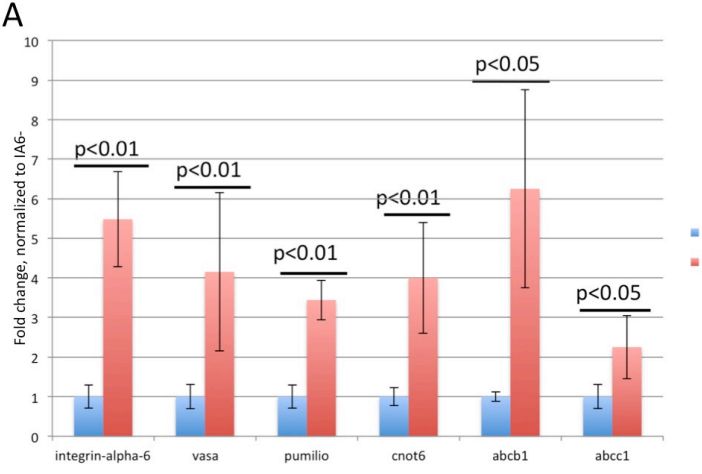

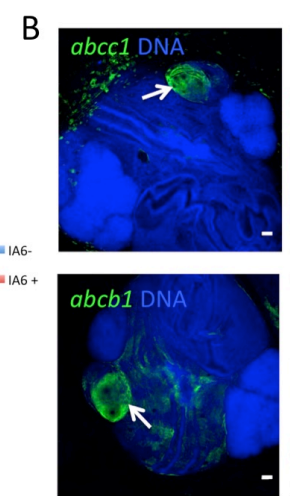

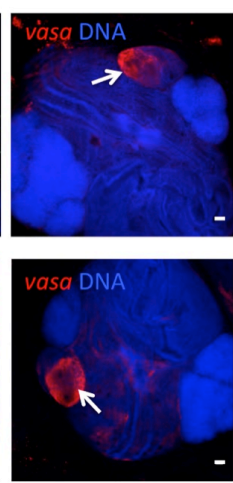

Figure 2: Botryllus germ cells express abcb1 and abcc1. A: Abcb1 and abcc1 are expressed in Integrin-alpha-6-positive (IA6+) germline stem cells. IA6-positive andnegative cells were isolated by flow cytometry and expression of germ cell marker genes (vasa, pumilio, cnot6) and ABC-transporters (abcb1 and abcc1) was assessed by quantitative real time PCR. Relative quantification was performed using the $2^{-\Delta \Delta C T}$. method, with actin as control gene. Data are expressed as averages of the relative expression ratio (fold change), normalized to IA6-negative cells. Standard deviations were calculated for each average expression ratio $(n=3)$. Statistical analysis was performed using Student's $t$-test. B: Representative examples of double-labeled fluorescent in situ hybridization showing expression of $a b c c 1$ and $a b c b 1$ in vasa-positive cells. All vasa-positive (red) germ cell precursors (arrows) co-express abcc1 and abcb1 (green, arrows). Red and green channels are shown individually with nuclear counterstaining (blue), and merged images on the right show co-expression of both genes (yellow) Scale bars $=20 \mu \mathrm{m}$.

\section{$A B C B 1$ and $A B C C 1$ activity is required for migration towards low concentrations of S1P}

To test whether inhibition of $A B C$ transporter activity affects migration of Botryllus germ cells we isolated IA6+ cells by flow cytometry and assessed their migratory activity to S1P in our transwell migration assay.

In the presence of inhibitors of either $A B C C 1$ or $A B C B 1$, migratory activity to a low concentration of S1P $(0.2 \mu \mathrm{M})$ is significantly reduced $(p \leq 0.05$, figure $3 A)$. An inhibitor of both $A B C C 1$ and $A B C B 1$ reduces migration even further, almost to control levels. In contrast, migration in the presence of a high concentration of S1P $(2 \mu \mathrm{M})$ is not significantly affected by $A B C$-transporter inhibition (Figure 3A).

These results suggest that $A B C C 1$ and $A B C B 1$ might export a secondary chemoattractant that enhances chemotaxis in the presence of low concentrations of the 
primary chemoattractant S1P (Figure 3B). In the presence of high concentrations of $\mathrm{S} 1 \mathrm{P}$, stimulation through the S1P receptor alone is sufficient to stimulate chemotaxis.
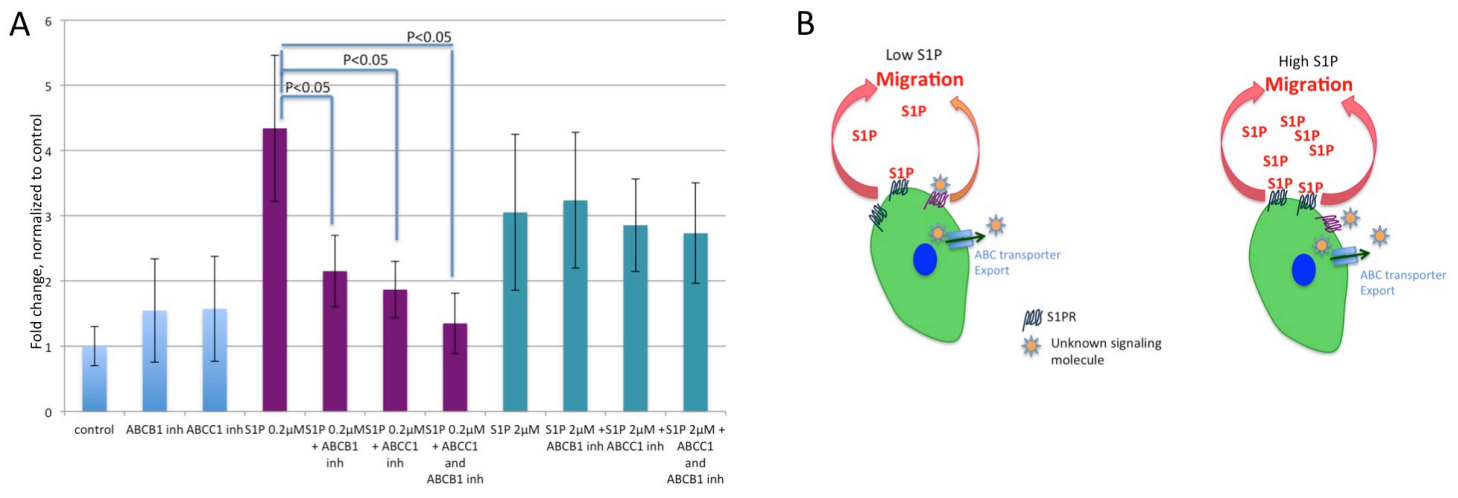

C

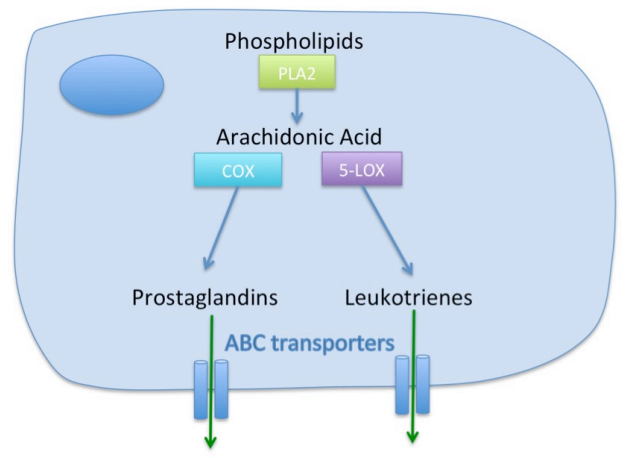

D

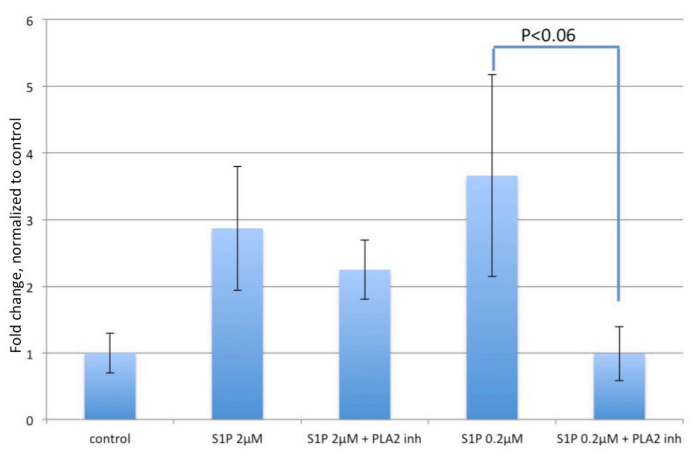

Figure 3: Migration of Integrin-alpha-6-positive germ cell precursors towards low concentrations of sphingosine-1-phosphate (S1P) depends on ABC-transporter activity. A) Migration assay of IA6-positive cells in response to different concentrations of S1P, with or without inhibitors of ABC-transporters, as indicated. No S1P was added to control wells. IA6+ cells were added to the upper chamber of a transwell system coated with laminin, and after $2 \mathrm{~h}$, migrated cells in the lower chamber were counted. Data are expressed as fold changes of numbers of migrated cells, normalized to unstimulated controls $(n=4)$. Statistical analysis was performed using Student's $t$-test. B: Hypothetical model of ABC-transporter mediated export of an unknown secondary chemoattractant signaling molecule. Migration to shallow gradients of S1P depends on ABC-transporter activity. Low-level stimulation of the S1P-receptor might induce production and $\mathrm{ABC}$-transporter mediated secretion of an unknown signaling molecule, which provides a secondary chemoattractant signal to enhance migration towards S1P. Steeper gradients of S1P are sufficient to stimulate chemotaxis, and ABC-transportermediated export of a secondary chemoattractant is not required. C: The cytoplasmic enzyme phospholipase A2 (PLA2) generates arachidonic acid from phospholipids. Arachidonic acid is further metabolized by either lipoxygenases (LOX) or Cyclooxygenases (COX) to generate bioactive lipids such as leukotrienes or prostaglandins, which are exported out of the cytoplasm by ABC transporters. $D$ : migration assay of IA6+ cells in response to S1P in the presence of an inhibitor of PLA2. Inhibition of PLA2 completely blocks the migratory activity to $0.2 \mathrm{uM}$ of S1P, but has no significant effect on migration towards $2 \mathrm{uM}$ of S1P. Data are expressed as fold 
changes of numbers of migrated cells, normalized to unstimulated controls $(n=4)$. Statistical analysis was performed using Student's t-test.

\section{Phospholipase A2 activity and lipoxygenase activity are required for migration to} S1P

Next, we aimed to assess for a possible secondary chemoattractant that might be exported by $A B C C 1$ and $A B C B 1$. $A B C$ transporters export a variety of substrates. Among these are a variety of lipid signaling molecules, such as phospholipids and derivatives of arachidonic acid ${ }^{11,16}$. In humans, the cytoplasmic enzyme phospholipase A2 (PLA2, Fig3C) generates the polyunsaturated omega-6 fatty acid arachidonic acid from phospholipids. Arachidonic acid is further metabolized by either lipoxygenases (LOX) or Cyclooxygenases (COX) to generate bioactive lipids such as leukotrienes or prostaglandins, which are exported out of the cytoplasm by $A B C$ transporters (Fig3C) ${ }^{16}$. To test whether a derivative of arachidonic acid plays a role in germ cell migration towards low concentrations of $\mathrm{S} 1 \mathrm{P}$, we assessed migratory activity in the presence of an inhibitor of PLA2. Inhibition of PLA2 completely blocked the migratory response to $0.2 \mu \mathrm{M}$ of S1P (Fig3D), but only had a mild effect on migration to a high concentration of S1P $(2 \mu \mathrm{M}, \mathrm{Fig} 3 \mathrm{D})$. These results show that a derivative of arachidonic acid is required for the migratory response to low concentrations of S1P. To assess whether this derivative is a product of cyclooxygenase or lipoxygenase, we tested the migratory response to $0.2 \mu \mathrm{M}$ of S1P in the presence of several inhibitors of COX-1 or COX-2 or an inhibitor of lipoxygenases. Botryllus schlosseri lipoxygenase (bslox) has homology to 5-LOX, 12LOX and 15-LOX from different organisms, and bslox expression is enriched in IA6+ cells (Figure 6A, fig S1C)). An inhibitor that blocks the activity of all 3 human lipoxygenases blocks germ cell migration to $0.2 \mu \mathrm{M}$ of S1P, whereas COX-inhibitors had no effect (Fig 4A). Furthermore, cyclooxygenases are not expressed at significant levels in IA6+ germ cells (Fig S1C). Migration to a high concentration of S1P $(2 \mu \mathrm{M})$ is not affected by LOX inhibition (Fig4A). These results show that an arachidonic-acid-derived product of lipoxygenase is exported by $A B C C 1$ or $A B C B 1$ and is required for migration towards low concentrations of S1P.

Inhibition of $A B C C 1, A B C B 1, P L A 2$ or Lipoxygenases reduces migration of germ cells to secondary bud niches in vivo. 
To test whether migration of germ cells to secondary buds in vivo requires activity of ABCC1, ABCB1, PLA2 or Lipoxygenase, we allowed B. schlosseri colonies to develop in the presence of inhibitors. Drugs were added to the seawater at stage $A 1$, when the new generation of zooids first begin to develop. Following the migration period, when the GSCs have migrated from old to new germline niches (Figure 1C), the colonies where fixed in formaldehyde and germ cell migration analyzed by vasa-FISH (Figure 4B). Vasapositive cells in the new niche were quantified using confocal microscopy. In colonies treated with inhibitors, significantly fewer vasa-positive cells (green) entered into new germline niche (white circles) ( $p \leq 0,01$, Figure $4 \mathrm{C}$ ) compared to vehicle treated controls.

A

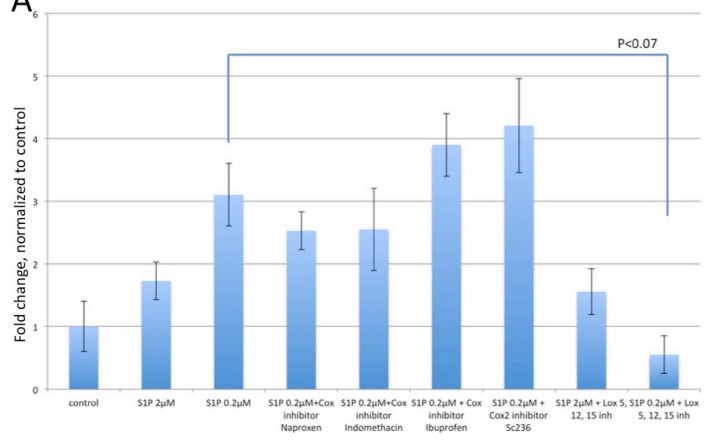

$C_{120}$

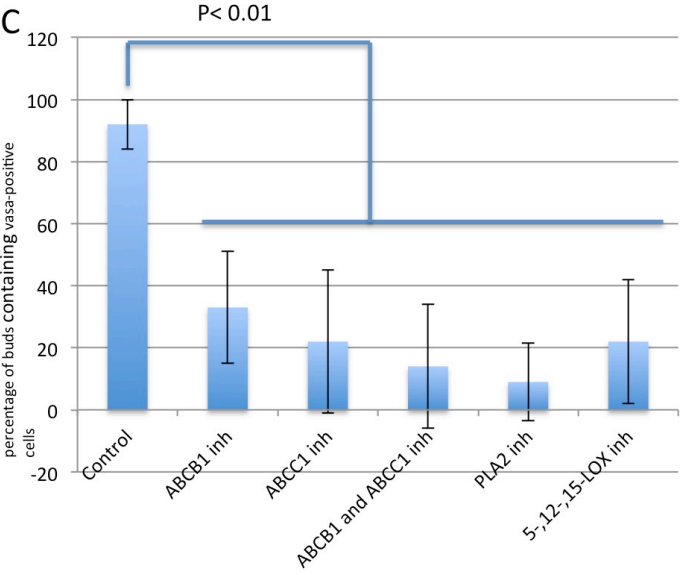

B
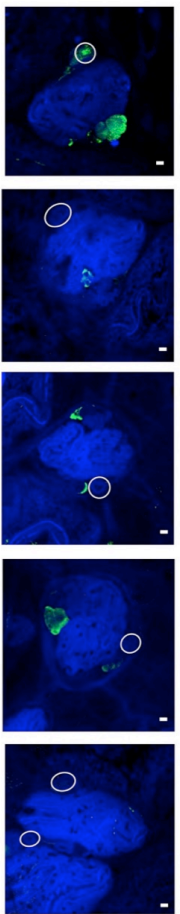
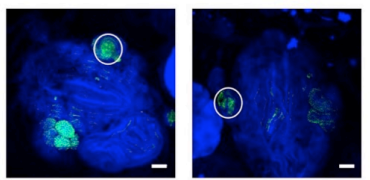

Control
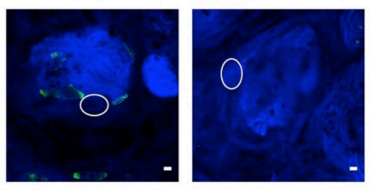

ABCB1 inh
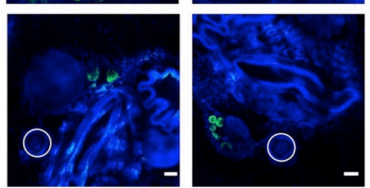

$\mathrm{ABCC1}$ inh
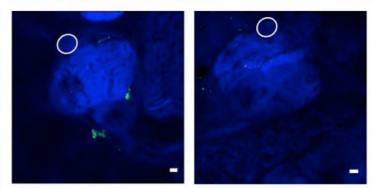

PLA2 inh

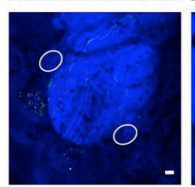

$5-, 12-, 15-$ LOX inh

Figure 4: Migration towards low concentrations of S1P depends on activity of Lipoxygenase. A: Migration assay of IA6+ cells in response to S1P, with or without inhibitors Cox1, Cox2 or 5-,12-, and 15-LOX, as indicated. Data are expressed as fold changes of numbers of migrated cells, normalized to unstimulated controls $(n=4)$. Statistical analysis was performed using Student's $t$-test. B and C: Homing of germ cells in vivo depends on activity of PLA2 and LOX. Animals were treated with inhibitors of $A B C$ transporters, PLA2 or 5-,12-, and 15-LOX for 3 days, starting at stage A1, and fixed at stage B2, when the secondary bud forms a closed double vesicle $(n=4)$. Controls were left untreated. Vasa-FISH was performed on fixed animals, and the number of secondary buds containing germ cells were counted by confocal microscopy. Nuclei 
were counterstained with Hoechst 33342 (blue). Scale bars $=20 \mu \mathrm{m}$. In control animals, vasa-positive germ cells (green) homed into the double vesicle stage secondary buds (circles). All 4 inhibitors significantly reduced migration of vasa-positive cells to secondary buds. Graph in C shows the percentage of double vesicle stage secondary buds containing vasa-positive cells for each treatment. Error bars represent the standard deviation for each average $(n=4)$. Statistical analysis was performed using Student's $t$ test.

\section{Botryllus germ cells express a receptor for the 12-LOX product 12-S-HETE}

Different types of lipoxygenases use arachidonic acid as a substrate to generate a variety of downstream signaling molecules, including leukotrienes, lipoxins, and other fatty acids such as different types of hydroperoxyeiocatetraenoic acid (HPETE) and hydroxyicosatetraenoic acid (HETE) (Figure 5A). To assess which lipoxygenase-product is responsible for enhancing migration to S1P, we tested an inhibitor of 5-LOX, as well as a cysteinyl leukotriene receptor antagonist. Neither of these significantly affected migration to S1P (Figure 5B). These data suggested that a product of either 12-LOX or 15-LOX is responsible for migration to S1P. 12-LOX generates 12-S-HETE, a signaling molecule that stimulates migration of cancer cells and smooth muscle cells ${ }^{17}$. In humans, the G-protein-coupled receptor GPR31 is a high affinity receptor for 12-S-HETE ${ }^{18}$. We identified a Botryllus homolog of GPR31 in our EST database, and have named it Bsgpr31. In humans, another receptor for 12-S-HETE, and for the 15-LOX product 15-SHETE is Leukotriene B4 receptor $2^{19}$, but we were not able to identify a homolog of this receptor in Botryllus. Using qPCR, we found that Bsgpr31 expression is significantly enriched in IA6+ cells and expressed at levels comparable to vasa (FigS1C and D). We also identified a Botryllus homolog of the closely related GPCR "Trapped in endoderm" tre-1, a fatty acid receptor that is important for germ cell guidance in Drosophila ${ }^{20}$. This receptor is not expressed in Integrin-alpha-6-positive cells (Fig S1C and D). Using in situ hybridization, we confirmed that Bsgpr31 mRNA is expressed exclusively in vasapositive cells migrating to secondary buds (Figure 5C, white arrows). Expression of $a b c c 1, a b c b 1$ or gpr31 does not show any significant changes during the blastogenic cycle in fertile and infertile animals in our published transcriptomes (Fig S2) ${ }^{21}$, indicating that these genes are always expressed in germ cells. This is in line with our own observation that migratory activity of IA6+ cells in vitro is independent of the blastogenic stage of the original colony. 
A

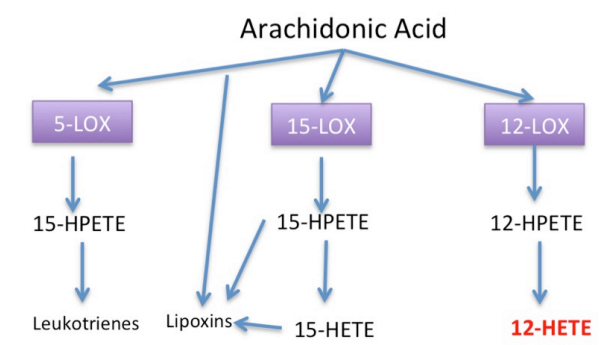

C

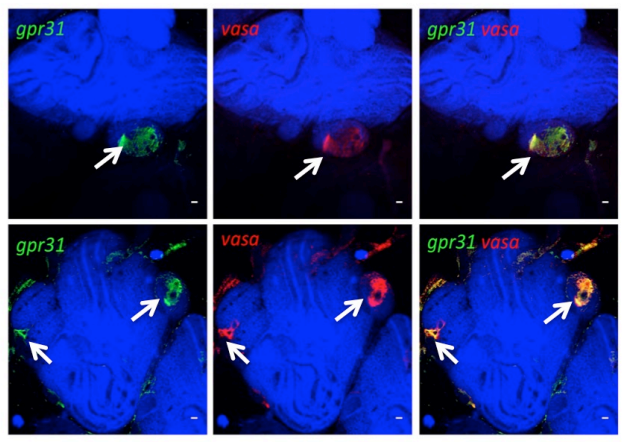

B

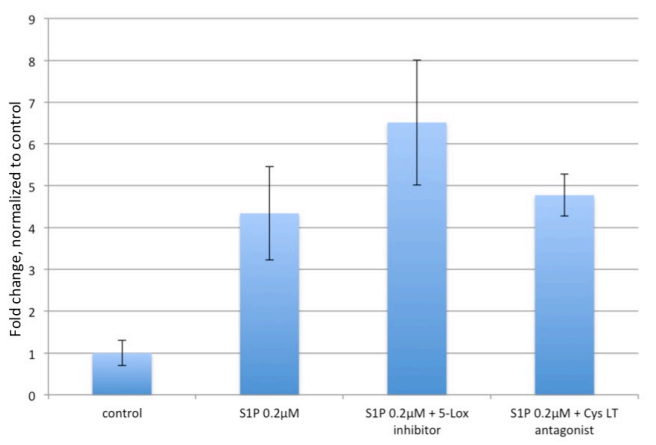

D

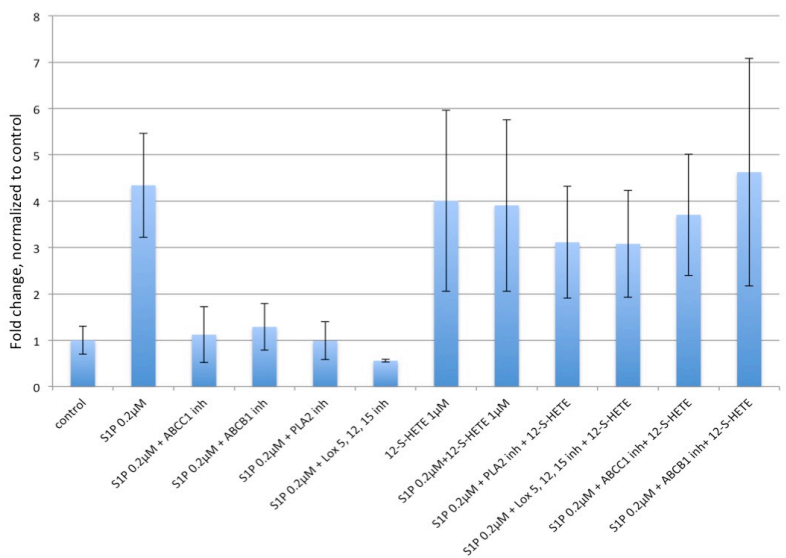

Figure 5: Botryllus germ cells express the 12-S-HETE receptor gpr31, 12-S-HETE rescues migration in the presence of $A B C$-transporter and lipoxygenase inhibitors. A: Migration assay of IA6-positive cells in response to $0.2 \mu \mathrm{M} \mathrm{S1P}$, with or without inhibitors 5-LOX or Cysteinyl Leukotriene Receptor, as indicated. Data are expressed as fold changes of numbers of migrated cells, normalized to unstimulated controls $(n=4)$. Statistical analysis was performed using Student's $t$-test. B: In humans, 3 different types of lipoxygenase metabolize arachidonic acid to various signaling agents. 12-Lox metabolizes arachidonic acid to 12(S)-hydroperoxy-5Z,8Z,10E,14Zeicosatetraenoic acid (12(S)-HpETE). 12(S)-HpETE is rapidly reduced to 12(S)-HETE by ubiquitous cellular peroxidases, such as Glutathione peroxidases. C: Representative examples of double-labeled fluorescent in situ hybridization showing expression of gpr31 in vasa-positive cells. All vasa-positive (red) germ cell precursors (arrows) co-express gpr31 (green, arrows). Red and green channels are shown individually with nuclear counterstaining (blue), and merged images on the right show co-expression of both genes (yellow) Scale bars $=20 \mu \mathrm{m}$. D: Migration assay of IA6-positive cells in response to $0.2 \mu \mathrm{M}$ S1P and/or 12-S-HETE, with or without inhibitors of ABC-transporters, PLA2 or 5-,12-, and 15-LOX, as indicated. Data are expressed as fold changes of numbers of migrated cells, normalized to unstimulated controls $(n=4)$. Statistical analysis was performed using Student's $t$-test.

The 12-LOX product 12-S-HETE stimulates germ cell migration and rescues inhibition of ABC-transporters, PLA2 and lipoxygenases. 
As vasa-positive cells in Botryllus express the putative receptor for 12-S-HETE, Bsgpr31, we aimed to test whether 12-S-HETE would stimulate migratory activity of IA6+ cells in vitro. 12-S-HETE alone has a stimulating effect on migration of IA6+ cells (Figure $5 \mathrm{D})$. 12-S-HETE induces migration nearly to nearly the same extent as $0.2 \mu \mathrm{M} \mathrm{S1P}$. In combination, both molecules in combination induce the same amount of migration as 12S-HETE alone. Importantly, adding exogenous 12-S-HETE rescues migratory activity to $0.2 \mu \mathrm{M}$ S1P in the presence of inhibitors of ABCC1, ABCB1, PLA2 or LOX (Figure 5D). These data suggest that migration to $0.2 \mu \mathrm{M}$ S1P is dependent on the secondary chemoattractant-12-S-HETE, and also demonstrates that the inhibitors used are not affecting chemotaxis non-specifically.

\section{2-S-HETE acts as a secondary chemoattractant and increases chemotaxis to S1P}

To test the effect of 12-S-HETE on S1P-induced chemotaxis, we characterized the migratory behavior of cells exposed to a chemotactic gradient in a 3D matrix. The chemotactic gradient was established by adding S1P and/or 12-S-HETE to the left reservoir of a chemotaxis chamber, and filtered seawater to the right reservoir, and cells were unmanipulated, or exposed to inhibitors of endogenous 12-S-HETE synthesis. We initially used identical concentrations of S1P and 12-S-HETE to those used in the transwell assays, and both the direction and average distance traveled by the GSCs under each condition are shown in Figure 6.

Initially, we characterized the response when a high concentration of S1P was added to the left reservoir (steep gradient, Figure 6A). Under these conditions, cells directionally migrate towards the left side (Figure 6A), and cover more distance then unstimulated controls (Figure 6B). This response required endogenous 12-S-HETE production, as inhibition of Lipoxygenase abolishes S1P-directed chemotaxis, and also reduced the total distance travelled (Figure 6B).

When 12-S-HETE and S1P are both added to the reservoir, cells cover more distance than in S1P alone, but loose directionality (Figure 6A, B). When 12-S-HETE alone was added to the left reservoir, cells migrate randomly (Figure 6A) and cover more distance than unstimulated controls (Figure 6B). We hypothesize that these results are due to the fact that arachidonic acid derived eicosanoids are very small molecules that diffuse rapidly, and upon release, the resulting gradients are shallow and transient. Cells that 
secrete such secondary chemoattractants must use a mechanism to create short-range gradients alone the cell axis ${ }^{22}$. In neutrophils responding to a primary gradient of fMLP, the secondary gradient is formed by localized secretion of $\mathrm{LTB}_{4}$ in exosomes to the region of the cell experiencing the highest concentration of fMLP. Our data suggest that when 12-S-HETE is added to the left reservoir it diffuses too quickly for a stable gradient to form, and the cells are exposed to 12-S-HETE from all sides and lose their ability to perform directional migration towards the primary chemoattractant S1P. Thus it appears that 12-S-HETE stimulates migration non-directionally if not in a gradient along the cell boundary.

We next attempted to create a shallow gradient of 12-S-HETE in the chemotaxis chamber by adding a very low concentration of 12-S-HETE to one of the corners of the left reservoir (pre-filled with seawater) immediately before live-imaging, so that 12-SHETE would diffuse more slowly towards the cells. Under these conditions (12-S-HETE shallow gradient), 12-S-HETE alone does not induce chemotaxis, but cells cover more distance than controls (Figure 6A, B). We next used the same technique to create a very shallow gradient of S1P. In this very shallow S1P gradient, cells cover more distance compared to controls, and even compared to cells migrating in a steep S1P gradient, but they are not able to migrate directionally towards S1P (Figure 6A, B). It has been shown that in suboptimal concentrations of chemoattractant, cells turn more frequently ${ }^{23}$, potentially facilitating the search for regions with optimal concentrations of chemoattractant. These results suggest that very low concentrations of S1P are not sufficient to stimulate production and secretion of 12-S-HETE.

However, when we used the technique described above to add a combined shallow gradient of 12-S-HETE and S1P to the corner of the reservoir, the cells were able to migrate directionally towards S1P, and covered more distance than in any of the other conditions tested (Figure 6A, B). 

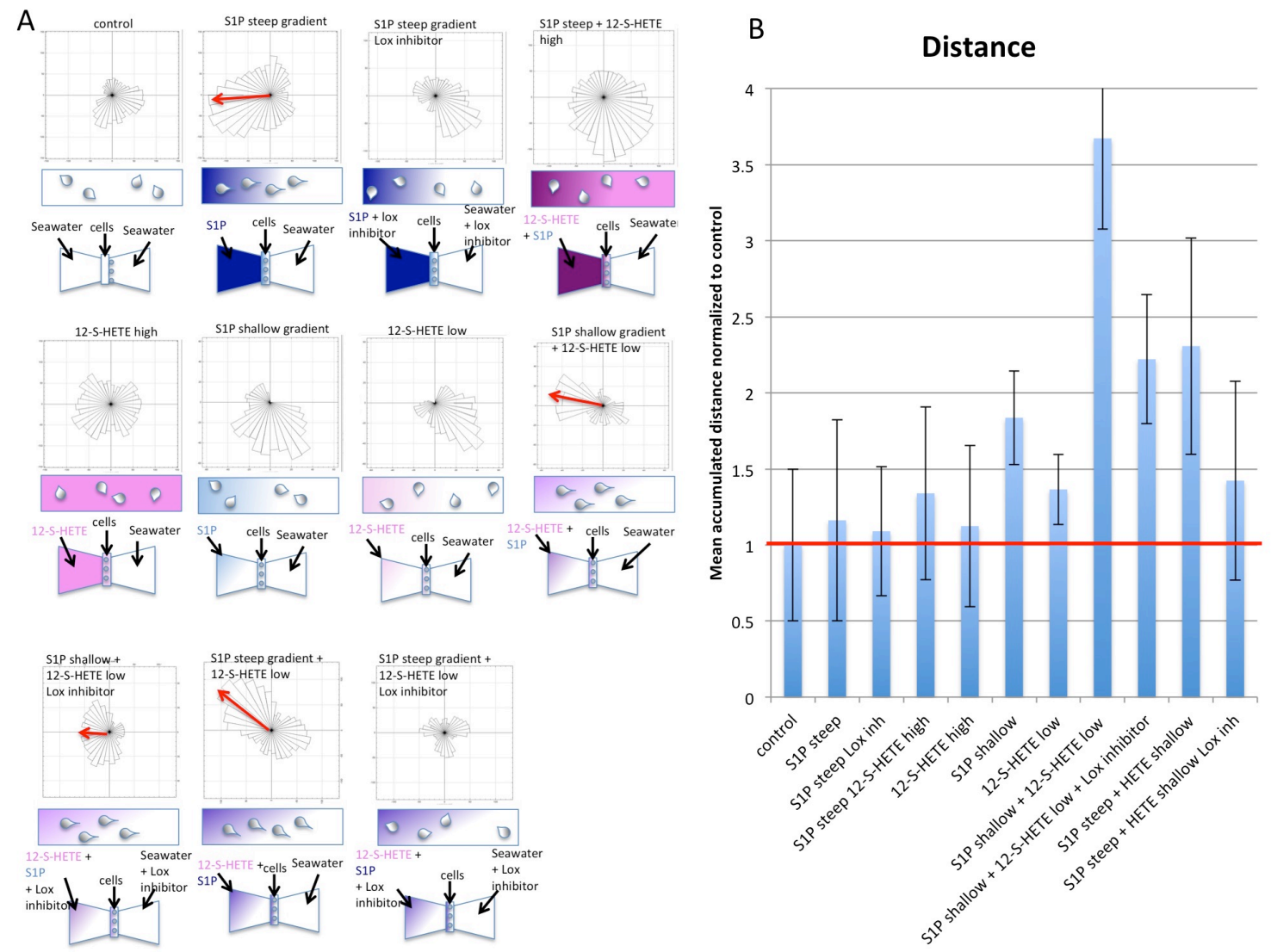

Figure 6: Migration to low concentrations of S1P depends on 12-S-HETE production: A: Chemotaxis assay. Chemotaxis was analyzed by live imaging of cells embedded in Matrigel in a chemotaxis chamber. The right reservoir contained filtered seawater, and for steep gradients the left reservoir contained 500nM 12-S-HETE (pink) or $0.2 \mu \mathrm{M}$ S1P (blue) or both. For shallow gradients, 50nM 12-S-HETE (light pink) or $0.2 \mu \mathrm{M}$ S1P (light blue) (final concentrations) were added to the indicated corner of the left reservoir. For controls, both reservoirs contained filtered seawater. Data from 3 independent experiments were combined and plotted as rose diagrams showing the directionality of cell paths for each condition tested. B: Average accumulated distance for cells migrating in each condition $(n=3)$, normalized to unstimulated controls, with standard deviation. The red line indicates the distance migrated by control cells (unstimulated).

This demonstrates that when present as a gradient, 12-S-HETE enhances chemotaxis to the primary chemoattractant S1P and acts as a secondary chemoattractant in Botryllus germ cells (illustrated in Figure 7A). Together, this data suggests that under normal conditions, detection of S1P induces 12-S-HETE secretion in a polarized manner, and that this signal relay mechanism is required for chemotaxis under physiological S1P concentrations. We next wondered if chemotaxis in a shallow exogenously applied gradient of S1P and 12-S-HETE also required autocrine production of 12-S-HETE. When a lipoxygenase inhibitor is added, chemotaxis and distance are reduced, 
indicating that autocrine production of 12-S-HETE is required for chemotaxis in cells migrating towards S1P (illustrated in Figure 7B), even in the presence of an external gradient of 12-S-HETE. This suggests the presence of a positive feedback loop, where autocrine production of 12-S-HETE is induced by extracellular gradients of S1P and 12S-HETE (illustrated in Figure 7A).

Together, this data suggests that under normal conditions, detection of S1P induces 12S-HETE secretion in a localized manner, and that this signal relay mechanism is required for chemotaxis under physiological S1P concentrations. Given that results using a high concentration of 12-S-HETE suggested that this molecule diffuses rapidly, we next wondered if chemotaxis in a shallow exogenously applied gradient of 12-S-HETE also required downstream, autocrine 12-S-HETE stimulation. In the next set of experiments cells treated with Lipoxygenase inhibitor were assayed under the same conditions (shallow S1P and shallow 12-S-HETE), and under these conditions chemotaxis and distance are reduced, indicating that autocrine production of 12-S-HETE is required for chemotaxis in cells migrating towards S1P (Figure 6; Illustrated in Figure 7B), even in the presence of an external shallow gradient of 12-S-HETE. This suggests the presence of a positive feedback loop, where autocrine production and localized secretion of 12-S-HETE is induced by extracellular gradients of S1P and 12-S-HETE (illustrated in Figure 7A). 

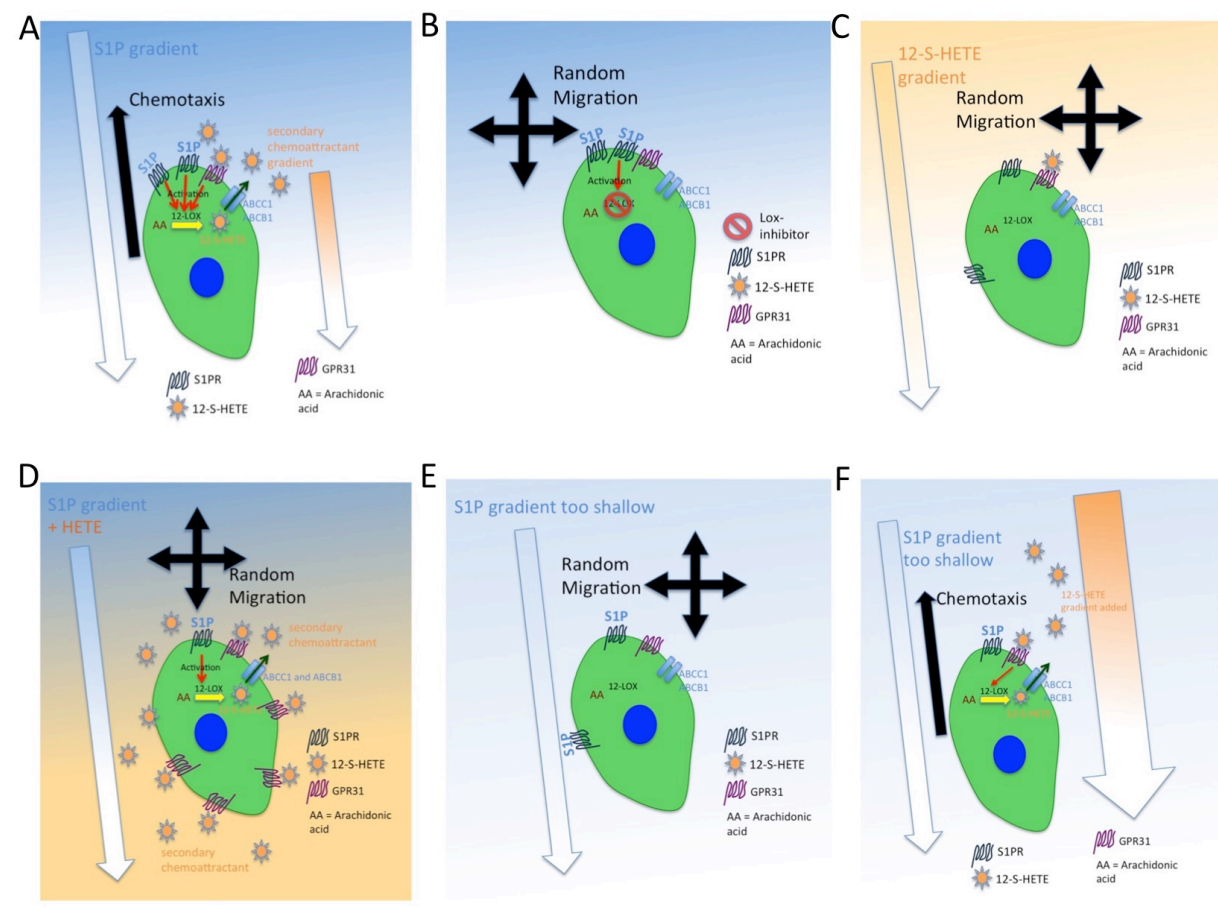

Figure 7: Hypothesized mechanism for 12-S-HETE acting a secondary chemoattractant during migration of germ cells towards S1P. A: In a gradient of S1P, S1P receptors are activated and stimulate activity of 12-LOX, leading to production and export of 12-S-HETE. 12-S-HETE acts as a secondary chemoattractant, and binds to GPR31 on the cell surface, resulting in enhanced chemotaxis. Activation of the 12-SHETE-receptor GPR31 stimulates activity of 12-LOX, sustaining chemotaxis. B: When 12-LOX is inhibited, no 12-S-HETE is secreted, resulting in lack of chemotaxis towards S1P and random migration. C: A gradient of 12-S-HETE alone does not stimulate chemotaxis, because the primary chemoattractant is missing. D: When 12-S-HETE is present in uniform concentration in all directions, cells lose their directionality, resulting in random migration. E: In sub-optimal concentrations of S1P or a gradient that is too shallow, S1P receptors are not sufficiently activated to stimulate secretion of 12-SHETE. F: When an artificial gradient of 12-S-HETE is added to a shallow gradient of S1P, chemotaxis towards S1P is stimulated. Activation of the 12-S-HETE-receptor GPR31 stimulates activity of 12-LOX, sustaining chemotaxis.

Interestingly, the same results were observed when a shallow gradient of 12-S-HETE is combined with a high concentration of S1P: chemotaxis towards S1P is enhanced and mean migration distance increases when combined with a shallow 12-S-HETE gradient. (Figure 6; illustrated in Figure 7A). However even under these high S1P concentrations, inhibition of lipoxygenase reduces chemotaxis and distance in this experiment, providing further evidence that an autocrine-feedback loop regulates 12-S-HETE secretion and 
may function to sustain chemotaxis in cells migrating towards S1P (illustrated in Figure $7 \mathrm{~A})$.

\section{Discussion}

This is the first report of an eicosanoid signaling molecule directing germ cell migration, and the first report of 12-S-HETE-stimulated migration of germ cells. We show that 12-SHETE acts as a secondary chemoattractant that facilitates chemotaxis of germ cells towards the primary chemoattractant S1P. This mechanism is termed signal relay, and it can extend the spatial range over which cells can be directed in a primary gradient. In exponential gradients, signal relay could potentially attract cells in areas where the slope of the primary gradient is shallow. For example, $\mathrm{LTB}_{4}$ enables directed migration of neutrophils when the primary gradient is too shallow to induce chemotaxis ${ }^{15}$. We show that migration of Botryllus germ cells towards low concentrations of S1P requires the activity of $A B C$-transporters and lipoxygenase (Illustration in Figure 7A, results in Figure 4, 5D and 6). Inhibition of LOX abolishes the ability of cells to directionally migrate towards S1P (Illustration in Figure 7A, results in Figure 6A). In a very shallow gradient of S1P, cells can only perform directed migration when a gradient of the 12-LOX product 12-S-HETE is present (Illustration in Figure 7E and F, results in Figure 6A), indicating that 12-S-HETE is a secondary chemoattractant that is required for directional migration towards the primary chemoattractant S1P. This idea is further supported by the fact that a gradient of 12-S-HETE alone does not induce chemotaxis (Illustration in Figure 7C, results in Figure 6).

Chemotaxis towards S1P is blocked by inhibition of Lox, even in the presence of an externally added gradient of 12-S-HETE, suggesting the presence of a positive feedback loop, where autocrine production of 12-S-HETE is induced by extracellular gradients of S1P and 12-S-HETE (illustrated in Figure 7A and F). When a higher concentration of 12S-HETE is added directly to one of the reservoirs of the chemotaxis chamber, cells migrate randomly (Illustration in Figure 7D), suggesting that a small molecule such as 12-S-HETE diffuses too quickly to form a stable gradient along the cell axis. The same phenomenon has been reported for other small fatty acid secondary chemoattractants, such as leukotriene B4 $\left(\mathrm{LTB}_{4}\right)^{22}$. Therefore, we hypothesize that the export and release of 12-S-HETE by migrating germ cells must be spatially and temporally controlled. In human neutrophils migrating towards a primary chemoattractant, secreted $\mathrm{LTB}_{4}$ is packaged in exosomes ${ }^{22}$ By gradually releasing $\mathrm{LTB}_{4}$, exosomes may prevent 
$\mathrm{LTB}_{4}$ profiles from rapidly reaching saturating concentrations ${ }^{15}$. If $12-\mathrm{S}-\mathrm{HETE}$ is packaged in such exosomes, $A B C$ transporters might be involved in controlling secretion of 12-S-HETE from exosomes. Alternatively, 12-S-HETE secretion could be spatially controlled along the axis of the migrating cell, and occur only at the leading edge, to form a gradient along the cell axis.

There is one main difference between chemotaxis in the 3D matrix and the transwell migration assay: there can be no stable gradient of 12-S-HETE in the transwell - this small molecule diffuses too quickly, and, therefore, the cells are likely sensing 12-SHETE from all directions. This may explain why there was no additive effect of S1P and 12-S-HETE in the transwell migration assay (Figure 5D), but in the 3D matrix, cells perform more chemotaxis and cover more distance in the presence of a gradient of 12S-HETE when it is added to a gradient of S1P (Figure $6 \mathrm{~A}, \mathrm{~B}$ ). In general, migration in a $3 D$ matrix is more physiological than migration on a $2 D$ plastic surface, so the results from the IBID assay are likely mimicking the in vivo situation more closely.

While $A B C$ transporters play known roles in dendritic cell and cancer cell migration ${ }^{16}$, only one study has so far reported a requirement of an $A B C$ transporter in germ cell migration. MDR49 regulates the export of farnesyl-modified mating factors in yeast and is expressed in the drosophila mesoderm. MDR49 mutants have defects in PGC migration ${ }^{12}$. Here, we show that in Botryllus, germ cell migration depends on activity of $A B C B 1$ and $A B C C 1$ and the export of an eicosanoid secondary chemoattractant. This is the first study suggesting a role for signal relay and secretion of a secondary chemoattractant in germ cells migrating in a primary chemotactic gradient. In addition, our data suggests that once a 12-S-HETE gradient is detected, cells amplify this signal via continued secretion to the region of the cell experiencing the highest concentration, initiating a positive feedback loop that would help drive and sustain chemotaxis. Polarized secretion could be due to redistribution of enzymes responsible for 12-S-HETE production, or the $A B C$ transporters required for secretion.

The roles of bioactive lipids in germ cell migration are poorly understood, but there is growing evidence for their importance. In Drosophila germ cell migration, the GPCR Tre1 directs migration through the midgut ${ }^{20}$. While the ligand for Tre1 still unknown, the closest mammalian homolog, GPR84, binds to medium chain fatty acids. In Drosophila and zebrafish, lipid phosphate phosphatases are required for directed migration of germ 
cells ${ }^{5,6}$. To our knowledge, no other studies have addressed the role of lipids in germ cell migration in other species. Here we show that the bioactive lipids S1P and 12-SHETE regulate germ cell migration in an invertebrate chordate, suggesting that bioactive lipids may play conserved roles in directing germ cell migration across phyla.

12-S-HETE has been shown to stimulate cell migration in human cell types. Specifically, it induces migration of cancer cells on laminin ${ }^{24}$. This is relevant since Botryllus germ cells also migrate on laminin ${ }^{7}$. 12-S-HETE induces PKC-dependent cytoskeletal rearrangements in tumor cells, resulting in increased motility ${ }^{17}$ and stimulates aortic smooth muscle cell migration ${ }^{25}$. Interestingly, upregulation of 12-LOX induces a migratory phenotype in cancer cells ${ }^{26}$, suggesting autocrine stimulation by $12-\mathrm{S}$-HETE plays a role in metastasis. In a carcinoma cell line, activation of beta- 4 integrin induces translocation of 12-LOX to the membrane and upregulates its enzymatic activity ${ }^{27}$. Finally, both 12-S-HETE and GPR31 expression positively correlate to prostrate cancer grade and progression in humans (Honn et al., 2016). In context of these observations, we hypothesize that in Botryllus germ cells, S1P-signaling and binding of Integrin-alpha6 to laminin might induce translocation of 12-LOX or ABC trannsporters to the leading edge, resulting in localized secretion of 12-S-HETE.

When a small amount of 12-S-HETE is added to a very shallow gradient of S1P, cells cover considerably more distance than with S1P alone (Figure 6B), suggesting that at the right concentration, 12-S-HETE not only enhances directional migration but also increases motility. Cells undergoing directional migration require environmental guidance cues as well as the ability to initiate and sustain motility. Depending on the organism, migrating germ cells must sustain directed migration for twenty-four to forty-eight hours ${ }^{3}$. In Botryllus, migration of germ cells to new, germline niches occurs during a defined $48 \mathrm{~h}$ period ${ }^{28}$. It is common for a migrating cell to require more than one signal to induce this type of directed migration and motility. In mice, the chemokine SDF-1 provides the guidance cue to migrating primordial germ cells, whereas signaling of SCF through the receptor c-kit enhances motility ${ }^{3}$. Our results suggest that a 12-S-HETE positive feedback response may be responsible for maintaining directional migration.

In conclusion, migration of GSC towards a shallow gradient of S1P depends on ABCtransporter mediated export of a secondary chemoattractant produced by lipoxygenase. The 12-lipoxygenase-product 12-S-HETE acts as a secondary chemoattractant and enhances directional migration towards shallow gradients of the primary chemoattractant 
S1P. In turn, our data suggest that 12-S-HETE must also be in a gradient, and moreover that exogenous12-S-HETE stimulation induces endogenous production, amplifying the secondary signal. We have discovered a novel mechanism of signal relay required for germ cell chemotaxis. Signal relay had been previously studied in neutrophils and Dictyostelium discoideum ${ }^{29}$, but to our knowledge has never been described in germ cells. Given the role of $12-\mathrm{S}-\mathrm{HETE}$ in cancer cell migration ${ }^{26}$, our study suggests that a conserved eicosanoid based signal relay mechanisms might operate in many other cell types across different species.

\section{Materials and Methods:}

Animals

Botryllus schlosseri colonies used in this study were lab-cultivated strains, spawned from animals collected in Santa Barbara, CA, and cultured in laboratory conditions at 18-20 ${ }^{\circ} \mathrm{C}$ according to ${ }^{30}$. Colonies were developmentally staged according to ${ }^{31}$.

\section{Cell Sorting}

Genetically identical, stage matched animals were pooled, and a single cell suspension was generated by mechanical dissociation. Whole animals were minced and passed through $70 \mu \mathrm{m}$ and $40 \mu \mathrm{m}$ cell strainers in ice-cold sorting buffer (filtered sea-water with $2 \%$ horse serum and 50mM EDTA). Anti-Human/Mouse-CD49f-eFluor450 (Ebioscience, cloneGoH3) was added at a dilution of 1/50 and incubated on ice for $30 \mathrm{~min}$ and washed with sorting buffer. Fluorescence activated cell sorting (FACS) was performed using a FACSAria (BD Biosciences) cell sorter. Samples were gated IA6 (CD49f)-positive or negative based on isotype control staining (RatlgG2A-isotype-control eFluor450, Ebioscience). Analysis was performed using FACSDiva software (BD Biosciences). Cells were sorted using a $70 \mu \mathrm{m}$ nozzle and collected into sorting buffer.

\section{Quantitative RT PCR}

Sorted cells were pelleted at $700 \mathrm{~g}$ for $10 \mathrm{~min}$, and RNA was extracted using the Nucleospin RNA XS kit (Macherey Nagel), which included a DNAse treatment step. RNA was reverse transcribed into cDNA using random primers (Life Technologies) and Superscript II Reverse Transcriptase (Life Technologies). Quantitative RT-PCR (Q-PCR) was performed using a LightCycler 480 II (Roche) and LightCycler DNA Master SYBR Green I detection (Roche) according to the manufacturers instructions. The 
thermocycling profile was $5 \mathrm{~min}$ at 95 , followed by 45 cycles of $95^{\circ} \mathrm{C}$ for $10 \mathrm{sec}, 60{ }^{\circ} \mathrm{C}$ for $10 \mathrm{sec}$. The specificity of each primer pair was determined by BLAST analysis (to human, Ciona and Botryllus genomes), by melting curve analysis and gel electrophoresis of the PCR product. To control for amplification of genomic DNA, 'no RT'-controls were used. Primer pairs were analyzed for amplification efficiency using calibration dilution curves. All genes included in the analysis had CT values of $<35$. Primer sequences are listed in Supplemental table 1. Relative gene expression analysis was performed using the $2^{-\Delta \Delta C T}$ Method. The CT of the target gene was normalized to the CT of the reference gene actin : $\Delta \mathrm{C}_{\mathrm{T}}=\mathrm{C}_{\mathrm{T} \text { (target) }}-\mathrm{C}_{\mathrm{T} \text { (actin). }}$ To calculate the normalized expression ratio, the $\Delta \mathrm{C}_{\mathrm{T}}$ of the test sample (IA6-positive cells) was first normalized to the $\Delta \mathrm{C}_{\mathrm{T}}$ of the calibrator sample (IA6-negative cells): $\Delta \Delta \mathrm{C}_{\mathrm{T}}=\Delta \mathrm{C}_{\mathrm{T} \text { (IA6-positive) }}-\Delta \mathrm{C}_{\mathrm{T} \text { (IA6-negative). }}$ Second, the expression ratio was calculated: $2^{-\Delta \Delta C T}=$ Normalized expression ratio. The result obtained is the fold increase (or decrease) of the target gene in the test samples relative to IA6-negative cells. Each GPCR was performed at least three times on cells from independent sorting experiments gene was analyzed in duplicate in each run. The $\Delta \mathrm{C}_{\mathrm{T}}$ between the target gene and actin was first calculated for each replicate and then averaged across replicates. The average $\Delta \mathrm{C}_{\mathrm{T}}$ for each target gene was then used to calculate the $\Delta \Delta C_{T}$ as described above. Data are expressed as averages of the normalized expression ratio (fold change). Standard deviations were calculated for each average normalized expression ratio $(n=6)$. Statistical analysis was performed using Student's T-test.

\section{In Situ Hybridization}

Whole mount in situ hybridization was performed as described in ${ }^{32}$. Briefly, B. schlosseri homologs of genes of interest were identified by tblastn searches of the $B$. schlosseri EST database (http://octopus.obs-vlfr.fr/public/botryllus/blast_ botryllus.php) using human or Ciona (when available) protein sequences. Primer pairs were designed to amplify a 500-800 bp fragment of each transcript (Primer sequences in Supplemental table 1). PCR was performed with Advantage cDNA Polymerase (Clontech, 639105) and products were cloned into the pGEM-T Easy vector (Promega, A1360). In vitro transcription was performed with SP6 or T7 RNA polymerase (Roche, 10810274001, 10881767001) using either digoxigenin or dinitrophenol labeling. HRP-conjugated antidigoxigenin antibody (Roche, 11207733910) or HRP-conjugated anti-dinitrophenol antibody (Perkin Elmer, FP1129) were used to detect labeled probes by fluorophore 
deposition (Fluorescein or Cyanine 3) using the TSA Plus System (Perkin Elmer, NEL753001KT). Nuclei were stained with Hoechst 33342 (Thermofisher). Imaging of labeled samples was performed using an Olympus FLV1000S Spectral Laser Scanning Confocal.

\section{Calcein assay of $A B C$ transporter activity}

Cells were isolated as described above for cell sorting and filtered through a $10 \mathrm{uM}$ cell strainer. Cells were incubated with Calcein-AM (1/1000, Thermo Fisher) in seawater for 30 minutes at room temperature in the presence of 10uM CP 1000356 hydrochloride (ABCB1-inhibitor), 10uM Probenecid (ABCB1 inhibitor) or 10uM Reversan (ABCB1 and $A B C C 1$ inhibitor). Controls were incubated in Calcein only. Unstained controls were incubated in seawater only. Live cells were gated using forward and side scatter properties. Mean fluorescence intensity $(\mathrm{MFI})$ of Calcein fluorescence was analyzed using a FACSAria (BD Biosciences) cell sorter and FACSDiva software (BD Biosciences).

\section{Transwell Migration Assay}

Transwell filters with $8 \mu \mathrm{m}$ pore size inserted in a 24 well plate (Corning) were coated with laminin over night at $4^{\circ} \mathrm{C}$ and briefly air dried before adding 50,000 sorted cells, resuspended in $100 \mu \mathrm{l}$ filtered seawater with $10 \%$ DMEM and $1 \% \mathrm{FBS}$. The bottom of the well contained filtered seawater with 10\% DMEM (Corning) /1\% FBS (Corning) and 1\%

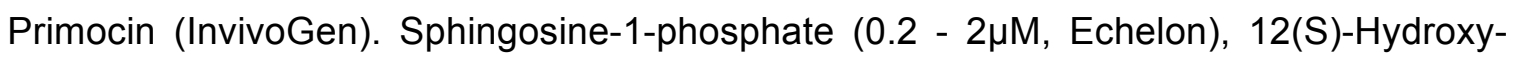
(5Z,8Z,10E,14Z)-eicosatetraenoic acid (80nM, Sigma-Aldrich) and 10uM CP 1000356 hydrochloride (ABCB1-inhibitor,), 10uM Probenecid (ABCB1 inhibitor), 10uM Reversan (ABCB1 and ABCC1 inhibitor), 10uM AACOCF3 (inhibitor of phospholipase A2), 0.5uM 2-TEDC (inhibitor of 5-,12- and 15-lipoxygenase), 10uM Zileuton (inhibitor of 5lipoxygenase), 1uM BAY-u 9773 (Cysteinyl leukotriene receptor antagonist), 10uM (S)(+)-Ibuprofen (Cox-1 inhibitor), 1mM SC 236 (Cox-2 inhibitor) (all from Tocris) were added to the bottom chamber as indicated. For controls, the bottom chamber contained filtered seawater with 10\% DMEM /1\% FBS and 1\% Primocin. After 2 hours incubation at room temperature, nuclei in the bottom well were stained with Hoechst $33342(1 / 1000$, Thermofisher) and manually counted. All assays were performed in triplicates with cells from 4 independent sorts. Statistical analysis was performed using Student's t-test. 
Small Molecule Inhibitor Treatment

Botryllus colonies were incubated in $5 \mathrm{ml}$ of seawater containing $25 \mu \mathrm{M}$ Reversan

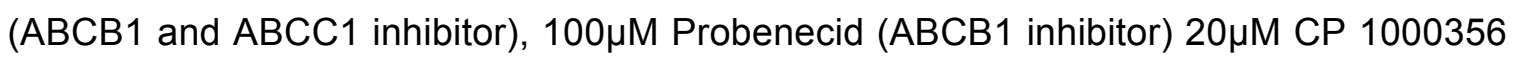
hydrochloride (ABCB1-inhibitor) or $25 \mu \mathrm{M}$ 2-TEDC (inhibitor of 5-,12- and 15lipoxygenase) or $14 \mu \mathrm{M}$ AACOCF3 (inhibitor of phospholipase A2). Controls were incubated in seawater. Treatment was started at stage A2, and animals were fixed at stage B2 and analyzed by in situ hybridization as described above. Each treatment was performed on 3 genetically identical colonies. Buds containing vasa-positive cells were counted on each treated and untreated colony under an epifluoresence microscope. Statistical analysis was performed using Student's t-test.

\section{Chemotaxis Assay}

Cells were isolated from the blood of Botryllus colonies, filtered through $10 \mu \mathrm{m}$ cell strainers and embedded in 50\% Matrigel (Corning) with 50\% filtered seawater. Whole blood was used to avoid the stress of cell sorting, since only vasa+ cells expressing S1PR $1^{33}$ or GPR31 (Figure 5C and S1C and D) will respond to stimulation with S1P or 12-S-HETE. $6 \mu$ l of gel were added to each chamber of an ibidi $\mu$-slide Chemotaxis (ibidi $\mathrm{GmbH}$, Martinsried, Germany). The gel was allowed to polymerize in a humidified chamber for 30 minutes at room temperature before adding filtered seawater to the right reservoir. The left reservoir was filled with filtered seawater containing Sphingosine-1phosphate $(0.2 \mu \mathrm{M}$, Echelon) or $12(\mathrm{~S})$-Hydroxy-(5Z,8Z,10E,14Z)-eicosatetraenoic acid (500nM, Sigma-Aldrich) or both. For samples containing 0.5uM 2-TEDC (inhibitor of 5,12- and 15-lipoxygenase), the inhibitor was added to both reservoirs. For controls, both reservoirs contained filtered seawater. To achieve a shallow gradient of S1P, $6 \mu \mathrm{l}$ of $2 \mu \mathrm{M}$ S1P were added to the corner of the left reservoir containing $60 \mu$ l of filtered seawater. To achieve a shallow gradient of 12-S-HETE, $6 \mu$ l of 50nM 12-S-HETE were added to the corner of the left reservoir containing $60 \mu$ l of filtered seawater. Live imaging was performed on a Leica SP8 confocal microscope at 15s intervals. Cell paths were tracked manually using the Manual Tracking Plugin in Image J. At least 30 cells were tracked in each field of view, and the data from 3 independent experiments were combined for the final analysis. Cell paths were analyzed using the Chemotaxis and Migration Tool Version 1.01 (https://ibidi.com/chemotaxis-analysis/171-chemotaxis-and-migrationtool.html) for Image J. 


\section{Acknowledgements:}

We would like to thank Amro Hamdoun for helpful discussions. Ben Lopez at the MCDB and NRI microscopy core facility is acknowledged for help with live imaging.

\section{Competing interests:}

The authors state no competing financial interests.

\section{Funding:}

\section{Eunice Kennedy Shriver National Institute of Child Health and Human Development (NICHD) HD092833 to AWD and SHK}

\section{References}

1 Vicente-Manzanares, M., Webb, D. J. \& Horwitz, A. R. Cell migration at a glance. J Cell Sci 118, 4917-4919, doi:10.1242/jcs.02662 (2005).

2 Richardson, B. E. \& Lehmann, R. Mechanisms guiding primordial germ cell migration: strategies from different organisms. Nat Rev Mol Cell Biol 11, 3749, doi:10.1038/nrm2815 (2010).

3 Barton, L. J., LeBlanc, M. G. \& Lehmann, R. Finding their way: themes in germ cell migration. Curr Opin Cell Biol 42, 128-137, doi:10.1016/j.ceb.2016.07.007 (2016).

4 Bieberich, E. It's a lipid's world: bioactive lipid metabolism and signaling in neural stem cell differentiation. Neurochem Res 37, 1208-1229, doi:10.1007/s11064-011-0698-5 (2012).

5 Starz-Gaiano, M., Cho, N. K., Forbes, A. \& Lehmann, R. Spatially restricted activity of a Drosophila lipid phosphatase guides migrating germ cells. Development 128, 983-991 (2001).

6 Paksa, A. et al. Repulsive cues combined with physical barriers and cell-cell adhesion determine progenitor cell positioning during organogenesis. Nat Commun 7, 11288, doi:10.1038/ncomms11288 (2016).

7 Kassmer SH, R. D., Langenbacher AD, Bui C, De Tomaso AW. Migration of germline progenitor cells is directed by sphingosine-1-phosphate signalling in a basal chordate. Nature Communications 6, doi:doi: 10.1038/ncomms9565 (2015).

8 Brown, F. D. et al. Early lineage specification of long-lived germline precursors in the colonial ascidian Botryllus schlosseri. Development 136, 3485-3494, doi:10.1242/dev.037754 (2009). 
9 Sabbadin, A. \& Zaniolo, G. Sexual Differentiation and Germ-Cell Transfer in the Colonial Ascidian Botryllus-Schlosseri. Journal of Experimental Zoology 207, 289-304, doi:DOI 10.1002/jez.1402070212 (1979).

10 Locher, K. P. Mechanistic diversity in ATP-binding cassette (ABC) transporters. Nat Struct Mol Biol 23, 487-493, doi:10.1038/nsmb.3216 (2016).

11 Neumann, J., Rose-Sperling, D. \& Hellmich, U. A. Diverse relations between ABC transporters and lipids: An overview. Biochim Biophys Acta Biomembr 1859, 605-618, doi:10.1016/j.bbamem.2016.09.023 (2017).

12 Ricardo, S. \& Lehmann, R. An ABC transporter controls export of a Drosophila germ cell attractant. Science 323, 943-946, doi:10.1126/science.1166239 (2009).

13 Randolph, G. J. Dendritic cell migration to lymph nodes: cytokines, chemokines, and lipid mediators. Semin Immunol 13, 267-274, doi:10.1006/smim.2001.0322 (2001).

14 Majumdar, R., Sixt, M. \& Parent, C. A. New paradigms in the establishment and maintenance of gradients during directed cell migration. Curr Opin Cell Biol 30, 33-40, doi:10.1016/j.ceb.2014.05.010 (2014).

15 Szatmary, A. C., Nossal, R., Parent, C. A. \& Majumdar, R. Modeling neutrophil migration in dynamic chemoattractant gradients: assessing the role of exosomes during signal relay. Mol Biol Cell 28, 3457-3470, doi:10.1091/mbc.E17-05-0298 (2017).

16 Fletcher, J. I., Haber, M., Henderson, M. J. \& Norris, M. D. ABC transporters in cancer: more than just drug efflux pumps. Nat Rev Cancer 10, 147-156, doi:10.1038/nrc2789 (2010).

17 Powell, W. S. \& Rokach, J. Biosynthesis, biological effects, and receptors of hydroxyeicosatetraenoic acids (HETEs) and oxoeicosatetraenoic acids (oxoETEs) derived from arachidonic acid. Biochim Biophys Acta 1851, 340-355, doi:10.1016/j.bbalip.2014.10.008 (2015).

18 Guo, Y. et al. Identification of the orphan G protein-coupled receptor GPR31 as a receptor for 12-(S)-hydroxyeicosatetraenoic acid. J Biol Chem 286, 33832-33840, doi:10.1074/jbc.M110.216564 (2011).

19 Yokomizo, T. Two distinct leukotriene B4 receptors, BLT1 and BLT2. J Biochem 157, 65-71, doi:10.1093/jb/mvu078 (2015).

20 Kunwar, P. S., Starz-Gaiano, M., Bainton, R. J., Heberlein, U. \& Lehmann, R. Tre1, a G protein-coupled receptor, directs transepithelial migration of Drosophila germ cells. PLoS Biol 1, E80, doi:10.1371/journal.pbio.0000080 (2003).

21 Rodriguez, D. et al. Analysis of the basal chordate Botryllus schlosseri reveals a set of genes associated with fertility. BMC Genomics 15, 1183, doi:10.1186/1471-2164-15-1183 (2014).

22 Majumdar, R., Tavakoli Tameh, A. \& Parent, C. A. Exosomes Mediate LTB4 Release during Neutrophil Chemotaxis. PLoS Biol 14, e1002336, doi:10.1371/journal.pbio.1002336 (2016). 
23 Wilkinson, P. C. Random locomotion; chemotaxis and chemokinesis. A guide to terms defining cell locomotion. Immunol Today 6, 273-278, doi:10.1016/0167-5699(85)90066-0 (1985).

24 Szekeres, C. K., Trikha, M., Nie, D. \& Honn, K. V. Eicosanoid 12(S)-HETE activates phosphatidylinositol 3-kinase. Biochem Biophys Res Commun 275, 690-695, doi:10.1006/bbrc.2000.3348 (2000).

25 Nakao, J., Ito, H., Chang, W. C., Koshihara, Y. \& Murota, S. Aortic smooth muscle cell migration caused by platelet-derived growth factor is mediated by lipoxygenase product(s) of arachidonic acid. Biochem Biophys Res Commun 112, 866-871 (1983).

26 Klampfl, T. et al. Up-regulation of 12(S)-lipoxygenase induces a migratory phenotype in colorectal cancer cells. Exp Cell Res 318, 768-778, doi:10.1016/j.yexcr.2011.12.017 (2012).

27 Tang, K. et al. Convergence of eicosanoid and integrin biology: 12lipoxygenase seeks a partner. Mol Cancer 14, 111, doi:10.1186/s12943-0150382-5 (2015).

28 Langenbacher, A. D. \& De Tomaso, A. W. Temporally and spatially dynamic germ cell niches in Botryllus schlosseri revealed by expression of a TGF-beta family ligand and vasa. Evodevo 7, 9, doi:10.1186/s13227-016-0047-5 (2016).

29 Garcia, G. L. \& Parent, C. A. Signal relay during chemotaxis. J Microsc 231, 529-534, doi:10.1111/j.1365-2818.2008.02066.x (2008).

30 Boyd HC, B. S., Harp JA, Weissman IL. Growth and sexual maturation of laboratory-cultured Monterey Botryllus schlosseri. Biological Bulletin 170, 91-109. (1986).

31 Lauzon, R. J., Ishizuka, K. J. \& Weissman, I. L. Cyclical generation and degeneration of organs in a colonial urochordate involves crosstalk between old and new: a model for development and regeneration. Dev Biol 249, 333348, doi:S0012160602907724 [pii] (2002).

32 Langenbacher, A. D., Rodriguez, D., Di Maio, A. \& De Tomaso, A. W. Wholemount fluorescent in situ hybridization staining of the colonial tunicate Botryllus schlosseri. Genesis, doi:10.1002/dvg.22820.

33 Kassmer, S. H., Rodriguez, D., Langenbacher, A. D., Bui, C. \& De Tomaso, A. W. Migration of germline progenitor cells is directed by sphingosine-1phosphate signalling in a basal chordate. Nat Commun 6, 8565, doi:10.1038/ncomms9565 (2015).

Figure Legends:

Figure 1: Morphology of Botryllus schlosseri colony, germ cell migration to secondary buds. 
A and B: colony morohology and asexual reproduction. All individual bodies within the colony are embedded in an extracellular matrix known as the tunic and share an extracorporeal vasculature. Adult zooids (blue outline) asexually reproduce by giving rise to primary (green outline) and secondary buds (red outline). C: Migration of germ cells to secondary buds. Secondary buds begin as small protrusions of the body wall of primary buds, and later form a closed double vesicle. This vesicle later grows and undergoes invaginations and tissue differentiation, completing development into the adult form. Germline stem cells and (GSC, $7-10 \mu \mathrm{M})$ and germ cell precursors $(30-50 \mu \mathrm{M})$ migrate from the primary bud into the secondary bud at the time when the double vesicle forms. When the double vesicle is fully formed, germ cells have completed migration. Migration into the secondary bud is directed by a chemotactic gradient of sphingosine-1phosphate, which is secreted within the secondary bud and detected by sphingosine-1phosphate-receptor-1 expressed by the migrating germ cells (Kassmer et al 2015).

Figure 2: Botryllus germ cells express abcb1 and abcc1. A: Abcb1 and abcc1 are expressed in Integrin-alpha-6-positive (IA6+) germline stem cells. IA6-positive andnegative cells were isolated by flow cytometry and expression of germ cell marker genes (vasa, pumilio, cnot6) and ABC-transporters (abcb1 and abcc1) was assessed by quantitative real time PCR. Relative quantification was performed using the $2^{-\triangle \triangle C T}$ method, with actin as control gene. Data are expressed as averages of the relative expression ratio (fold change), normalized to IA6-negative cells. Standard deviations were calculated for each average expression ratio $(n=3)$. Statistical analysis was performed using Student's t-test. B: Representative examples of double-labeled fluorescent in situ hybridization showing expression of abcc1 and abcb1 in vasa-positive cells. All vasa-positive (red) germ cell precursors (arrows) co-express abcc1 and abcb1 (green, arrows). Red and green channels are shown individually with nuclear counterstaining (blue), and merged images on the right show co-expression of both genes (yellow) Scale bars $=20 \mu \mathrm{m}$.

Figure 3: Migration of Integrin-alpha-6-positive germ cell precursors towards low concentrations of sphingosine-1-phosphate (S1P) depends on ABC-transporter activity. A) Migration assay of IA6-positive cells in response to different concentrations of S1P, with or without inhibitors of ABC-transporters, as indicated. No S1P was added to control wells. IA6+ cells were added to the upper chamber of a transwell system 
coated with laminin, and after $2 \mathrm{~h}$, migrated cells in the lower chamber were counted. Data are expressed as fold changes of numbers of migrated cells, normalized to unstimulated controls $(n=4)$. Statistical analysis was performed using Student's $t$-test. B: Hypothetical model of ABC-transporter mediated export of an unknown secondary chemoattractant signaling molecule. Migration to shallow gradients of S1P depends on ABC-transporter activity. Low-level stimulation of the S1P-receptor might induce production and $\mathrm{ABC}$-transporter mediated secretion of an unknown signaling molecule, which provides a secondary chemoattractant signal to enhance migration towards S1P. Steeper gradients of S1P are sufficient to stimulate chemotaxis, and ABC-transportermediated export of a secondary chemoattractant is not required. C: The cytoplasmic enzyme phospholipase A2 (PLA2) generates arachidonic acid from phospholipids. Arachidonic acid is further metabolized by either lipoxygenases (LOX) or Cyclooxygenases (COX) to generate bioactive lipids such as leukotrienes or prostaglandins, which are exported out of the cytoplasm by ABC transporters. $D$ : migration assay of IA6+ cells in response to S1P in the presence of an inhibitor of PLA2. Inhibition of PLA2 completely blocks the migratory activity to $0.2 \mathrm{uM}$ of S1P, but has no significant effect on migration towards $2 \mathrm{uM}$ of S1P. Data are expressed as fold changes of numbers of migrated cells, normalized to unstimulated controls $(n=4)$. Statistical analysis was performed using Student's $t$-test.

Figure 4: Migration towards low concentrations of S1P depends on activity of Lipoxygenase. A: Migration assay of IA6+ cells in response to S1P, with or without inhibitors Cox1, Cox2 or 5-,12-, and 15-LOX, as indicated. Data are expressed as fold changes of numbers of migrated cells, normalized to unstimulated controls $(n=4)$. Statistical analysis was performed using Student's $t$-test. B and C: Homing of germ cells in vivo depends on activity of PLA2 and LOX. Animals were treated with inhibitors of $A B C$ transporters, PLA2 or 5-,12-, and 15-LOX for 3 days, starting at stage A1, and fixed at stage B2, when the secondary bud forms a closed double vesicle $(n=4)$. Controls were left untreated. Vasa-FISH was performed on fixed animals, and the number of secondary buds containing germ cells were counted by confocal microscopy. Nuclei were counterstained with Hoechst 33342 (blue). Scale bars $=20 \mu \mathrm{m}$. In control animals, vasa-positive germ cells (green) homed into the double vesicle stage secondary buds (circles). All 4 inhibitors significantly reduced migration of vasa-positive cells to secondary buds. Graph in C shows the percentage of double vesicle stage secondary 
buds containing vasa-positive cells for each treatment. Error bars represent the standard deviation for each average $(n=4)$. Statistical analysis was performed using Student's $t$ test.

Figure 5: Botryllus germ cells express the 12-S-HETE receptor gpr31, 12-S-HETE rescues migration in the presence of $A B C$-transporter and lipoxygenase inhibitors. A: Migration assay of IA6-positive cells in response to $0.2 \mu \mathrm{M} \mathrm{S1P}$, with or without inhibitors 5-LOX or Cysteinyl Leukotriene Receptor, as indicated. Data are expressed as fold changes of numbers of migrated cells, normalized to unstimulated controls $(n=4)$. Statistical analysis was performed using Student's $t$-test. B: In humans, 3 different types of lipoxygenase metabolize arachidonic acid to various signaling agents. 12-Lox metabolizes arachidonic acid to 12(S)-hydroperoxy-5Z,8Z,10E,14Zeicosatetraenoic acid (12(S)-HpETE). 12(S)-HpETE is rapidly reduced to $12(\mathrm{~S})$-HETE by ubiquitous cellular peroxidases, such as Glutathione peroxidases. C: Representative examples of double-labeled fluorescent in situ hybridization showing expression of gpr31 in vasa-positive cells. All vasa-positive (red) germ cell precursors (arrows) co-express gpr31 (green, arrows). Red and green channels are shown individually with nuclear counterstaining (blue), and merged images on the right show co-expression of both genes (yellow) Scale bars $=20 \mu \mathrm{m}$. D: Migration assay of IA6-positive cells in response to $0.2 \mu \mathrm{M}$ S1P and/or 12-S-HETE, with or without inhibitors of ABC-transporters, PLA2 or 5-,12-, and 15-LOX, as indicated. Data are expressed as fold changes of numbers of migrated cells, normalized to unstimulated controls $(n=4)$. Statistical analysis was performed using Student's $t$-test.

Figure 6: Migration to low concentrations of S1P depends on 12-S-HETE production: A: Chemotaxis assay. Chemotaxis was analyzed by live imaging of cells embedded in Matrigel in a chemotaxis chamber. The right reservoir contained filtered seawater, and for steep gradients the left reservoir contained 500nM 12-S-HETE (pink) or $0.2 \mu \mathrm{M} \mathrm{S1P}$ (blue) or both. For shallow gradients, 50nM 12-S-HETE (light pink) or $0.2 \mu \mathrm{M}$ S1P (light blue) (final concentrations) were added to the indicated corner of the left reservoir. For controls, both reservoirs contained filtered seawater. Data from 3 independent experiments were combined and plotted as rose diagrams showing the directionality of cell paths for each condition tested. B: Average accumulated distance for cells migrating in each condition $(n=3)$, normalized to unstimulated controls, with 
standard deviation. The red line indicates the distance migrated by control cells (unstimulated).

Figure 7: Hypothesized mechanism for 12-S-HETE acting a secondary chemoattractant during migration of germ cells towards S1P. A: In a gradient of S1P, S1P receptors are activated and stimulate activity of 12-LOX, leading to production and export of 12-S-HETE. 12-S-HETE acts as a secondary chemoattractant, and binds to GPR31 on the cell surface, resulting in enhanced chemotaxis. Activation of the 12-SHETE-receptor GPR31 stimulates activity of 12-LOX, sustaining chemotaxis. B: When 12-LOX is inhibited, no 12-S-HETE is secreted, resulting in lack of chemotaxis towards S1P and random migration. C: A gradient of 12-S-HETE alone does not stimulate chemotaxis, because the primary chemoattractant is missing. D: When 12-S-HETE is present in uniform concentration in all directions, cells lose their directionality, resulting in random migration. $\mathrm{E}$ : In sub-optimal concentrations of S1P or a gradient that is too shallow, S1P receptors are not sufficiently activated to stimulate secretion of 12-SHETE. F: When an artificial gradient of 12-S-HETE is added to a shallow gradient of S1P, chemotaxis towards S1P is stimulated. Activation of the 12-S-HETE-receptor GPR31 stimulates activity of 12-LOX, sustaining chemotaxis. 